\title{
Probiotics VSL\#3 are effective in reversing non-alcoholic steatohepatitis in a mouse model
}

\author{
Prasant Kumar Jena ${ }^{1 \#}$, Lili Sheng ${ }^{1 \#}$, Yongchun $\mathrm{Li}^{1,2,3}$, Yui-Jui Yvonne Wan ${ }^{1}$ \\ ${ }^{1}$ Department of Pathology and Laboratory Medicine, University of California, Davis, Sacramento, CA, USA; ${ }^{2}$ Department of Gastroenterology, \\ Zhujiang Hospital, Southern Medical University, Guangzhou 510282, China; ${ }^{3}$ Department of Infectious Diseases, Nanhai Hospital, Southern \\ Medical University, Foshan 528200, China \\ Contributions: (I) Conception and design: YJY Wan; (II) Administrative support: None; (III) Provision of study materials or patients: None; (IV) \\ Collection and assembly of data: PK Jena, L Sheng, Y Li; (V) Data analysis and interpretation: All authors; (VI) Manuscript writing: All authors; (VII) \\ Final approval of manuscript: All authors. \\ \#These authors contributed equally to this work. \\ Correspondence to: Yu-Jui Yvonne Wan, PhD. Department of Pathology and Laboratory Medicine, University of California, Davis Health Systems, \\ Room 3400B, Research Building III, 4645 2nd Ave, Sacramento, CA 95817, USA. Email: yjywan@ucdavis.edu.
}

Background: Probiotic VSL\#3 is used to treat ulcerative colitis. This study examines the effect of VSL\#3 in non-alcoholic steatohepatitis (NASH) that has liver carcinogenic potential.

Methods: Western diet (WD)-fed wild-type (WT) mice that do not have hepatic inflammation with lymphocyte infiltration and carcinogenic potential were used for baseline comparison. Age-, sex-, and dietmatched bile acid (BA) receptor farnesoid X receptor (FXR) knockout (KO) mice, which developed severe NASH and had the potential for liver cancer development, were supplemented with and without VSL\#3 for 7 months. All the mice were euthanized when they were 10 months old.

Results: Supplementation with VSL\#3 completely abolished hepatic lymphocyte infiltration, reduced hepatic fat content, and improved insulin sensitivity in WD-fed FXR KO mice. In addition, VSL\#3 normalized dysregulated BA homoeostasis by inhibiting the classical BA synthesis pathway, inducing the alternative BA pathway, and activating ileal G-protein coupled BA receptor 1 (GPBAR1)-regulated signaling. Moreover, VSL\#3 reconstructed the gut microbiota by reducing Bacteroidaceae, Porphyromonadaceae, and Helicobacteraceae as well as increasing Lachnospiraceae. Further, VSL\#3 enriched the abundance of Ruminococcus and Faecalibacterium, which generate butyrate, at the genus level. It also increased the copy number of the butyrate-producing genes $b c 0 A$ and $b u k$, suggesting their anti-inflammatory and metabolic effects.

Conclusions: VSL\#3 is useful in reversing NASH that occurred due to dysregulated BA synthesis and dysbiosis, suggesting its potential in liver cancer prevention.

Keywords: Bile acid (BA); farnesoid X receptor (FXR); G-protein coupled bile acid receptor-1 (GPBAR1); microbiota; inflammation.

Submitted May 15, 2019. Accepted for publication Sep 09, 2019.

doi: 10.21037/hbsn.2019.09.07

View this article at: http://dx.doi.org/10.21037/hbsn.2019.09.07

\section{Introduction}

Through the gut-liver axis, the liver is constantly exposed to gut-derived components. This work tests a hypothesis that probiotics, which are effective in treating intestinal diseases such as ulcerative colitis, are also useful in treating a severe form of non-alcoholic steatohepatitis (NASH) that has tumorigenic potential.

The probiotic VSL $\# 3{ }^{\circledR}$ is prescribed for ulcerative colitis treatment (1). In animal models, VSL\#3 protects against hepatic NASH in dextran sulfate sodium-treated $A p o E^{-/-}$ mice and high fat diet-fed young rats as well as ob/ob mice (2-4). However, in methionine-choline-deficient (MCD) 
diet-induced NASH, VSL\#3 fails to prevent liver steatosis and inflammation but ameliorates hepatic fibrosis (5). It is important to note that the ketogenic high-fat diet is used for weight loss in humans, and the MCD diet improves insulin sensitivity in rodents $(6,7)$. Thus, the effects of VSL\#3 in NASH treatment warrants further investigation using human-relevant animal models.

Bile acid (BA) receptor FXR knockout (KO) mice, which have elevated BAs, develop steatosis, NASH, and liver cancer spontaneously (8-11). Similarly, patients who have hepatic cirrhosis and liver cancer have reduced FXR, making FXR-deficient mice human-relevant animal models (12). Furthermore, BAs are produced by converting hepatic cholesterol using both hepatic and gut microbial enzymes and thus can be used to explain how nutrients, through gut microbes, affect metabolism and inflammatory signaling. Therefore, we studied the impact of VSL\#3 on regulating BA receptor signaling as well as gut microbiota to elucidate the potential underlying effects of VSL\#3.

Our published data showed that WD-fed FXR KO male mice had the most serious NASH and the greatest potential for liver carcinogenesis compared with WD-fed wildtype (WT) mice or healthy diet-fed FXR KO mice of both sexes (8). Hence, we used WD-fed FXR KO male mice to test the effect of VSL\#3. Sex-, diet-, and age-matched WT mice, which do not have the potential for liver cancer development, were used for baseline comparisons. Our data revealed that VSL\#3 was very effective in preventing the hepatic lymphocyte infiltration that occurred in WDfed FXR KO mice. Moreover, VSL\#3 reduced hepatic cholesterol and triglyceride to levels that were lower than those found in WD-fed WT mice. Additionally, the health benefits of VSL\#3 were accompanied by normalized expression of BA homeostasis genes, increased G-protein coupled BA receptor 1 (GPBAR1)-regulated signaling, and reconstructed gut microbiota community structure. Moreover, the data also uncovered bacteria that were linked with the development and resolution of NASH. Taken together, this study not only shows the effectiveness of probiotics in reversing NASH but also suggests their potential to prevent liver cancer.

\section{Methods}

\section{Mice and VSL\#3 supplementation}

Specific pathogen-free C57BL/6 WT mice (Jackson Laboratory, Sacramento, CA, USA) and FXR KO mice (13) were housed in separated steel microisolator cages at $22{ }^{\circ} \mathrm{C}$ with a 12-hour light/dark cycle. Both genotypes were fed a WD containing $21.2 \%$ fat, $34 \%$ sucrose, and $0.2 \%$ cholesterol (Envigo, Indianapolis, IN, USA) after weaning (3 weeks old, 6-10 mice per group). For interventions, 3-month-old WD-fed FXR KO male mice were given VSL\#3 containing four Lactobacillus species, Lactobacillus paracasei, L. plantarum, L. acidophilus, and L. delbrueckii subspecies bulgaricus, as well as three Bifidobacterium species including B. longum, B. lactis, and B. breve, plus Streptococcus thermophiles $\left(10^{9}\right.$ cfu per mouse, orally, once a week, Lot: number: 05150542, Sigma-Tau Pharmaceutics, Inc., Gaithersburg, MD, USA) in saline while mice continued on a WD for an additional 7 months. All the mice were euthanized when they were 10 months old. Experiments were conducted in accordance with the National Institutes of Health Guidelines for the Care and Use of Laboratory Animals under protocols approved by the Institutional Animal Care and Use Committee of the University of California, Davis.

\section{Insulin tolerance test}

After fasting $6 \mathrm{~h}$, mice were given insulin intraperitoneally (1 U/kg body weight, Sigma-Aldrich) to conduct insulin (ITT) tolerance tests. Glucose levels were measured at 0 , $15,30,60,90$, and $120 \mathrm{~min}$ post insulin injection by the OneTouch Ultra 2 (Johnson \& Johnson, New Brunswick, NJ, USA) using the tail. The area under the curve (AUC) of the blood glucose levels over time was calculated.

\section{Biochemical analysis and histology}

Serum alanine aminotransferase (ALT; Pointe Scientific, Canton, MI, USA), alkaline phosphatase (ALP; Pointe Scientific, Canton, MI, USA), and hepatic triglyceride and cholesterol (BioAssay Systems, Hayward, CA, USA) levels were quantified according to the manufacturer's instructions. Hematoxylin and eosin-stained liver sections were subjected to histology analysis. Steatosis score was graded on a scale of $0(<5 \%), 1(5-33 \%), 2(34-66 \%)$, and $3(>66 \%)$, and hepatic lymphocyte infiltration score was graded on a scale of 0 (absent), 1 (rare), 2 (mild), 3 (moderate), and 4 (severe).

\section{Quantification of BAs}

Hepatic and serum BAs were quantified based on published 

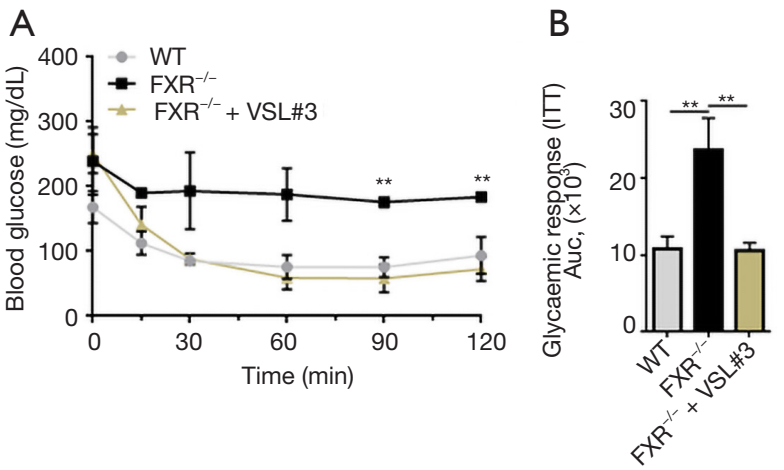

Figure 1 The effects of VSL\#3 on insulin sensitivity in WDfed FXR KO mice. (A) Blood glucose level based on insulin tolerance test; (B) area under curve of blood glucose level post insulin injection of age-, sex-, and diet-matched WT and FXR KO mice supplemented with and without VSL\#3 for 7 months. Data expressed as mean \pm SD. $\mathrm{n} \geq 6$ per group. ${ }^{* *}, \mathrm{P}<0.01$. WD, Western diet; WT, wild-type; FXR, farnesoid X receptor; KO, knockout.

methods $(8-10,14)$. The detection of BAs was carried out on a Prominence ${ }^{\mathrm{TM}}$ UFLC system (Shimadzu, Kyoto) coupled to an API 4000 QTRAP mass spectrometer (AB Sciex, Redwood City, CA, USA) operated in the negative ionization mode. Chromatography was performed on a Kinetex C18 column $(50 \mathrm{~mm} \times 2.1 \mathrm{~mm}, 2.6 \mu \mathrm{m})$ maintained at $40{ }^{\circ} \mathrm{C}$ preceded by a high-pressure column prefilter. The mobile phase consisted of a gradient of methanol delivered at a flow rate of $0.4 \mathrm{~mL} / \mathrm{min}$. Mass Spectrometer parameters were described in our previous publication (15).

\section{Gene expression profiling}

RNA was isolated using TRIzol (Invitrogen, Carlsbad, CA, USA) and reverse transcribed into cDNA. The qRTPCR was performed on an ABI 7900HT real-time PCR system using Power SYBR Green PCR Master Mix (Applied Biosystems, Foster City, CA, USA). The mRNA levels were normalized to the level of Gapdh mRNA.

\section{Quantification of bacterial DNA and 16S rRNA gene sequencing}

For bacterial gene quantification, DNA was extracted from cecal content (0.05 gram) using ZR Fecal DNA MiniPrep Kit (Zymo Research, Irvine, CA, USA), quantified by NanoDrop (Thermo Scientific, Wilmington, DE, USA), and amplified using primers based on the published sequences (16-18). A dissociation step was included to analyze the melting profile of the amplified products. In parallel, qPCR was done using ten-fold serial diluted synthetic DNA fragments of a bacterial gene with known concentrations (Integrative DNA technologies, Redwood city, CA, USA). Bacterial DNA concentration was calculated using standard curves of diluted synthetic DNA fragment based on published methods $(14,19,20)$.

Illumina sequencing of barcoded 16S rRNA gene amplicons of genomic DNA was performed based on published methods $(14,21)$. Variable region 4 of the $16 \mathrm{~S}$ rRNA gene was amplified and sequenced. DNA sequence reads were demultiplexed and classified with a custom python application, dbcAmplicons (https://github.com/ msettles/dbcAmplicons), to identify and assign reads by both expected barcode and primer sequences (20). The Ribosomal Database Project Bayesian classifier was used to assign sequences to phylotypes (22). Reads were assigned to the first Ribosomal Database Project taxonomic level with a bootstrap score $\geq 50$.

\section{Statistical analysis}

Data are expressed as mean \pm SD. Differences between groups at microbiota family and genus levels were calculated by the Kruskal-Wallis test. All other comparisons were calculated by two-tailed Student's $t$-test or one-way ANOVA followed by Tukey's test using GraphPad Prism 6 software (GraphPad Software, La Jolla, CA, USA). P values were adjusted for multiple comparisons using false discovery rate. $\mathrm{P}<0.05$ was considered statistically significant.

\section{Results}

\section{The effect of VSL\#3 on insulin sensitivity}

When diet- and sex-matched WT and FXR KO mice were 10-month old, FXR KO mice developed insulin resistance. However, VSL\#3 supplementation reversed it, and there was no difference in insulin sensitivity between $\mathrm{WT}$ and VSL\#3 supplemented FXR KO mice (Figure 1).

\section{The effect of VSL\#3 on hepatic steatosis and inflammation}

WD-fed FXR KO mice had increased fat scores and hepatic lymphocyte infiltration compared to WD-fed WT mice, and VSL\#3 improved hepatic fat scores and completely prohibited hepatic lymphocyte infiltration in WD-fed FXR 

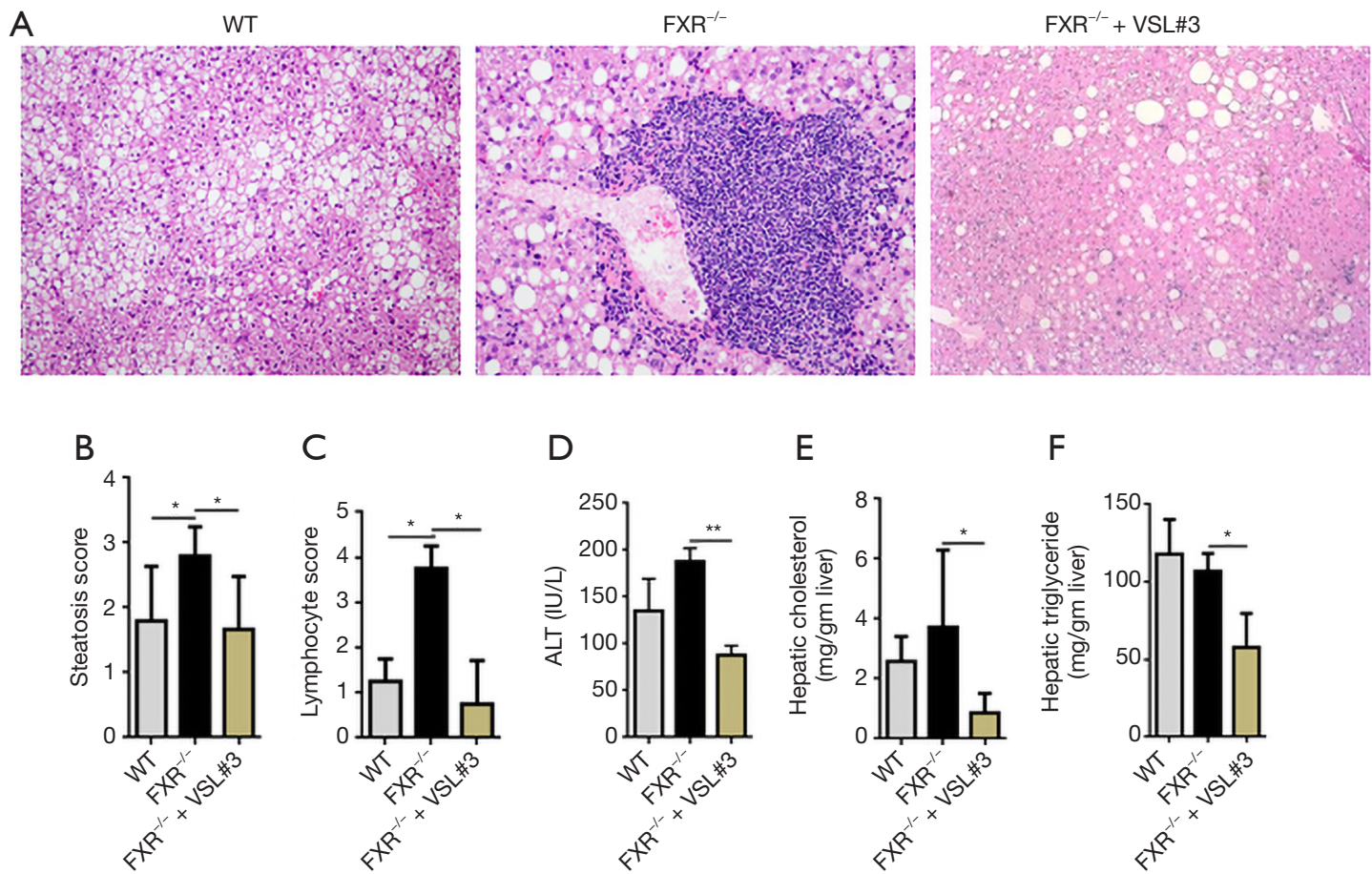

C

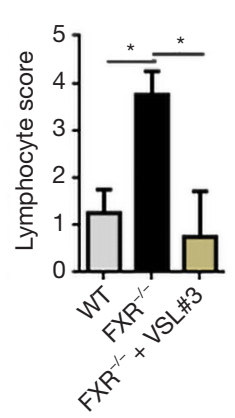

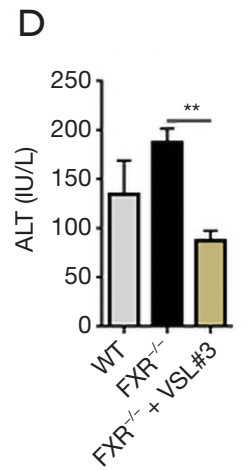
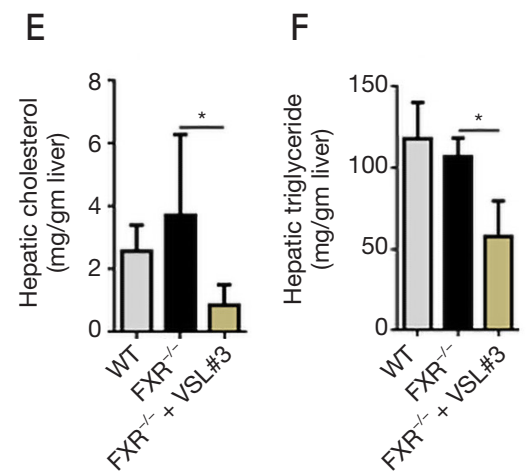

Figure 2 The effects of VSL\#3 in WD-fed FXR KO mice. Hematoxylin and eosin stain (HE, x40) of liver (A), hepatic steatosis score (B), hepatic lymphocyte score (C), serum ALT (D), hepatic cholesterol (E), and hepatic triglyceride (F) levels of WD-fed WT mice and WDfed FXR KO mice supplemented with and without VSL\#3 for 7 months. Data expressed as mean $\pm \mathrm{SD}$. $\mathrm{n} \geq 6$ per group. *, $\mathrm{P}<0.05$, **, $\mathrm{P}<0.01$. WD, Western diet; WT, wild-type; FXR, farnesoid X receptor; KO, knockout; ALT, alanine transferase.

KO mice (Figure $2 A, B, C$ ). In addition, VSL\#3 reduced serum ALT, as well as hepatic cholesterol and triglyceride (Figure 2D,E,F). In addition, FXR deficiency increased hepatic Ill $\beta, I l 6$, Tnf $\alpha, C c l 17$, Il4, and Foxp 3 mRNA levels, which were reduced by VSL\#3 supplementation (Figure $3 A$ ). Likewise, increased ileal $I l 1 \beta, I l 6$, Tnf $\alpha$, and $C c l 17$ mRNA levels due to lack of FXR were reduced by VSL\#3 supplementation (Figure 3B). In addition, ileal mRNA levels of Il10, Reg3r, and tight junction genes Occludin and Claudin 1 were decreased due to lack of FXR and induced by VSL\#3 (Figure 3B). Taken together, VSL\#3 effectively reversed hepatic inflammation in WD-fed FXR KO mice.

\section{The impact of VSL\#3 on BA profile}

Dysregulated BA synthesis accounts for liver carcinogenesis in FXR KO mice (23). We investigated the effect of probiotics VSL\#3 in altering BA profiles (Figure 4). FXRdeficient mice had increased hepatic $\alpha$-, and $\beta$-muricholic acid ( $\alpha$ MCA, $\beta$ MCA), taurodeoxycholic acid (TDCA), taurochenodeoxycholic acid (TCDCA), deoxycholic acid
(DCA), and hyodeoxycholic acid (HDCA), and VSL\#3 supplementation reversed those changes (Figure 4A). In addition, VSL\#3 consistently increased hepatic FXR agonists, such as cholic acid (CA) and chenodeoxycholic acid (CDCA), and GPBAR1 ligands, such as taurolithocholic acid (TLCA) and lithocholic acid (LCA), as well as ursodeoxycholic acid (UDCA). Moreover, serum levels of CA, CDCA, and TCA were reduced with FXR deficiency, and VSL\#3 reversed those changes. Serum UDCA level increased with FXR inactivation and was further increased by VSL\#3 (Figure 4B).

\section{The effect of VSL\#3 on BA synthesis}

FXR inactivation induced cholesterol $7 \alpha$-hydroxylase (Cyp7a1) and sterol 12 $\alpha$-hydroxylase (Cyp8b1), while VSL\#3 supplementation reduced them at mRNA and protein levels (Figure $5 A, B$ ). Additionally, FXR KO mice had reduced ileal FGF15 protein level, and VSL\#3 increased it (Figure 5B). Thus, VSL\#3 may inhibit BA synthesis by increasing ileal FGF15. In addition, VSL\#3 induced the expression of 

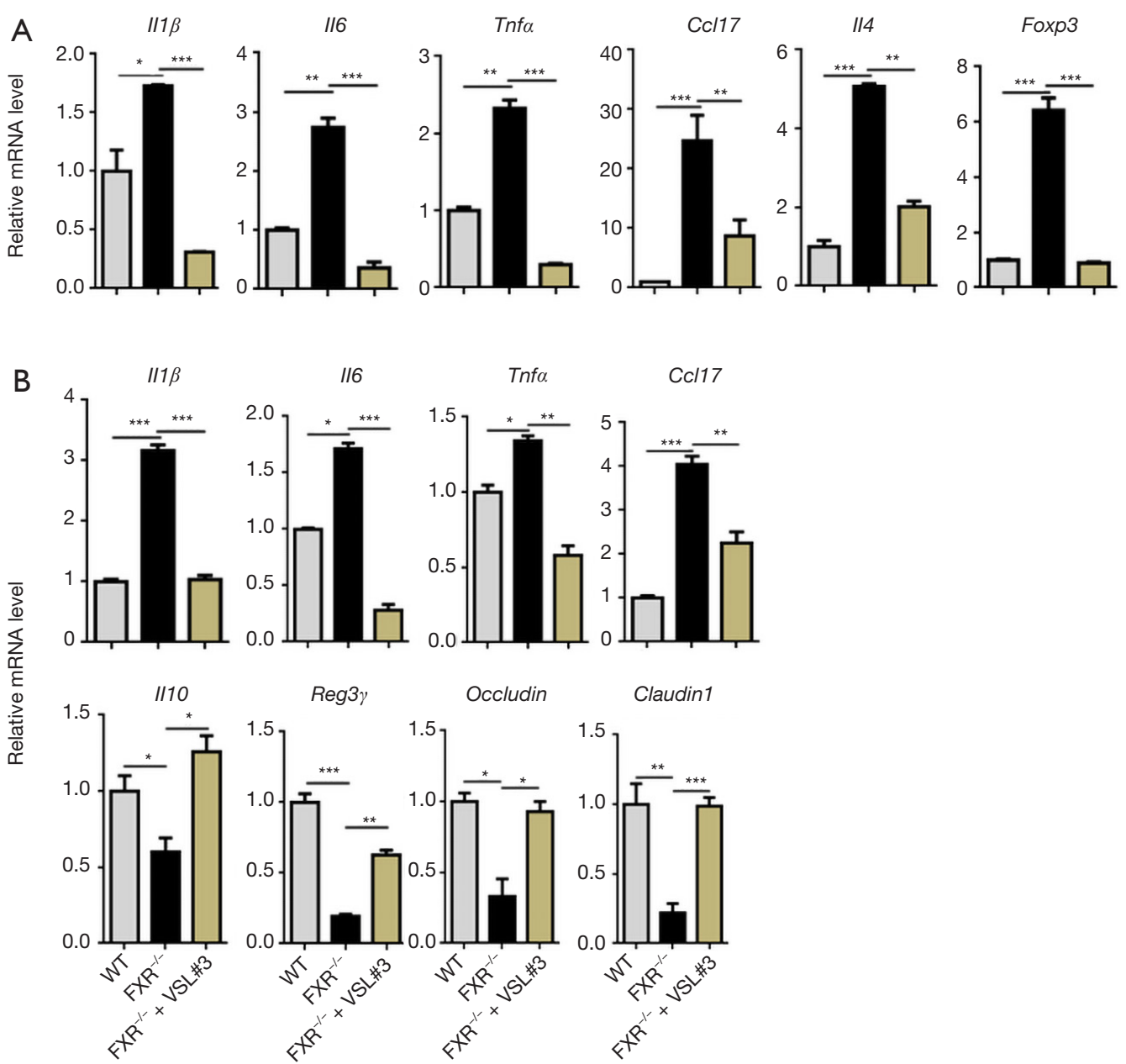

Figure 3 The expression of genes implicated in the inflammatory signaling. Hepatic (A) and ileal (B) gene expression in WD-fed WT and WD-fed FXR KO mice supplemented with and without VSL\#3 for 7 months. Data are expressed as mean \pm SD. $\mathrm{n} \geq 6$ per group. *, P<0.05, **, $\mathrm{P}<0.01$, *** $\mathrm{P}<0.001$. WD, Western diet; WT, wild-type; FXR, farnesoid X receptor; KO, knockout.

25 -hydroxycholesterol $7 \alpha$-hydroxylase $(C y p 7 b 1)$ and sterol 27-hydroxylase (Cyp27a1) genes that encode enzymes involved in an alternate pathway of BA synthesis. Moreover, VSL\#3 increased hepatic oxysterol $7 \alpha$-hydroxylase 2 (Cyp39a1), sodium/BA cotransporter (Ntcp), and organic anion-transporting polypeptides (Oatp1b2), which were all reduced due to lack of FXR. Furthermore, hepatic SULT2A1 was increased by VSL\#3 at mRNA and protein levels, suggesting increased sulfation (Figure $5 A, B$ ). Major BA organic and steroid transporters such as Ost $\alpha$, Ost $\beta$, and apical sodium-dependent BA transporter $(A s b t)$ were reduced in the ileum of FXR KO mice, and VSL\#3 reversed those changes, which might lead to increased BA transport (Figure 5C).

FXR KO and VSL\#3 supplementation also affect BA production that uses bacterial enzymes. WD-fed FXR KO mice had increased copy numbers of secondary BAgenerating bacterial gene baif and BA de-conjugation gene $b s h$ in the cecum. VSL\#3 reduced the copy number of the baif and bsh, suggesting reduced secondary BA production as well as deconjugation (Figure 5D).

\section{The effect of VSL\#3 on GPBAR1 signaling}

Lack of FXR also reduced the expression of GPBAR1, which is consistent with published data (24), and VSL\#3 supplementation induced ileal GPBAR $1 \mathrm{mRNA}$ and protein levels in FXR KO mice (Figure 6A,B). In addition, VSL\#3 increased GBPAR1-regulated signaling genes that include $G l p 1 r$ (glucagon like peptide 1 receptor), $P c 1 / 3$ 

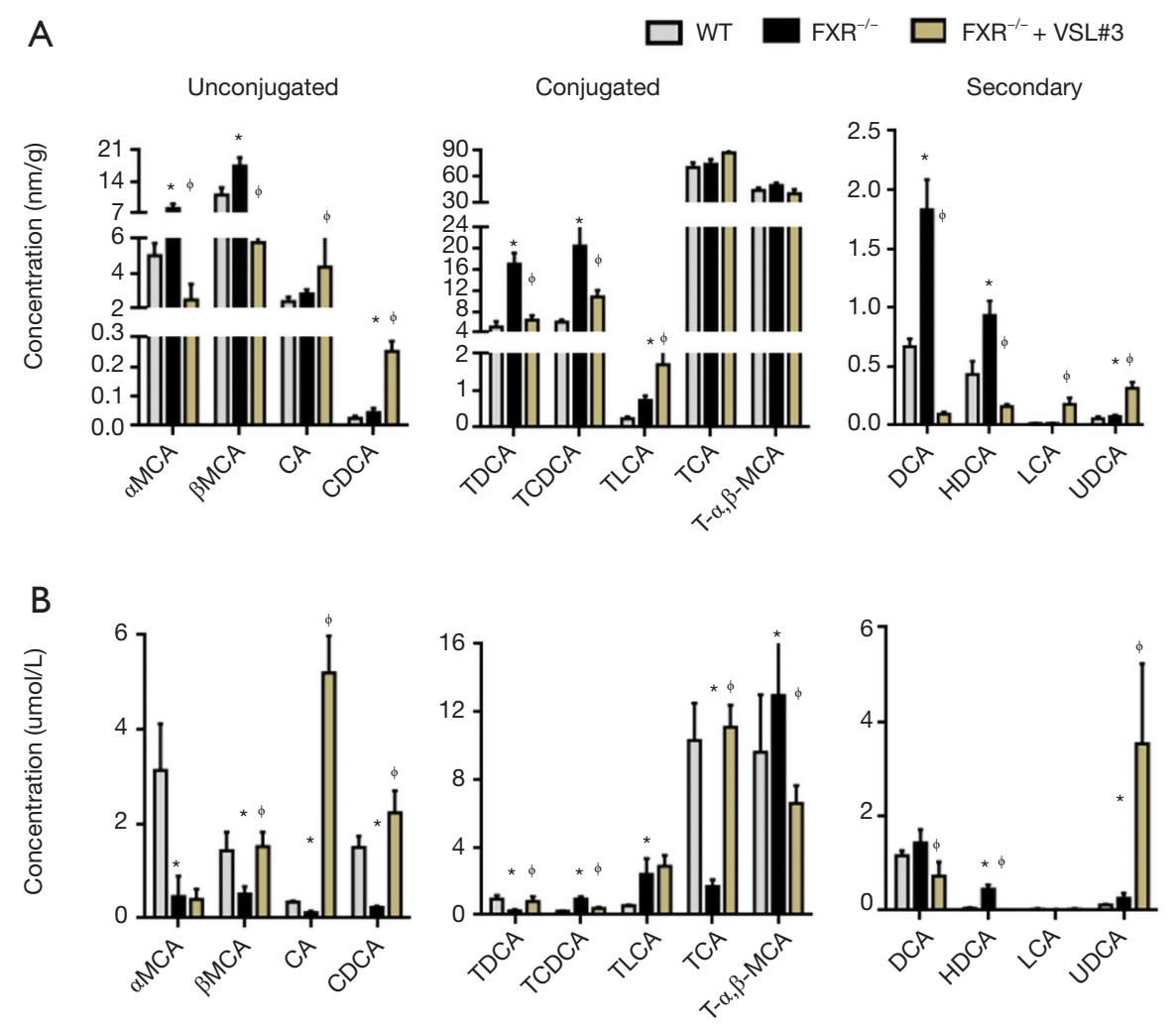

Figure 4 The effects of VSL\#3 on hepatic and serum BA profiles. Hepatic (A) and serum (B) BA profiles. Data are expressed as mean \pm SD. $\mathrm{n} \geq 6$ per group. *, $\mathrm{P}<0.05$ (WT mice compared with FXR KO mice), and ${ }^{\phi}, \mathrm{P}<0.05$ (FXR KO mice compared with VSL\#3 supplementedFXR KO mice). WT, wild-type; FXR, farnesoid X receptor; KO, knockout.

(proprotein convertase 1 and 3), Gcg (preproglucagon), Dio2 (iodothyronine Deiodinase 2), Nos1 (nitric oxide synthase 1), and Nos3 (nitric oxide synthase 3 ) in the ileum (Figure $6 A$ ). Thus, VSL\#3 reversed the suppressed GPBAR1 signaling pathways found in WD-fed FXR KO mice.

\section{The effect of VSL\#3 on gut microbiota community}

FXR inactivation increased the abundance of genus Bilophila wadsworthia and bacterial gene morA (methyl coenzyme-M reductase) as well as $d s r A$ (dissimilatory sulfite reductase), encoding the enzymes for methane and hydrogen sulfide production, respectively. However, VSL\#3 reversed these changes (Figure 7A). Moreover, VSL\#3 supplementation reversed the reduced abundance of butyrate-producing genes including $b c o A$ (butyryl-CoA: acetate CoA-transferase) and $b u k$ (butyrate kinase) in FXR KO mice.

Cecal microbiota analysis using 16s rRNA sequencing revealed that WT and FXR KO mice with or without VSL\#3 supplementation had different microbiome profiles
(Figure $7 B$ ). Under the Bacteroidetes phylum, FXR KO mice had increased Bacteroidaceae and Porphyromonadaceae families, which were reduced by VSL\#3 (Figure 7B). In addition, the abundance of the Rikenellaceae family, which was reduced by FXR KO, was further reduced by VSL\#3 (Figure 7B). Within the Firmicutes phylum, the abundance of butyrate producing bacterial families such as Clostridiaceae, Lachnospiraceae, and Erysipelotrichaceae was reduced by FXR deficiency, and VSL\#3 increased the abundance of Lachnospiraceae. Under the Actinobacteria phylum, the relative abundance of Coriobacteriaceae, which modulates intestinal cholesterol absorption and host metabolism, was reduced by FXR inactivation, but VSL\#3 did not change it $(25,26)$ (Figure $7 B)$. Importantly, under the Proteobacteria phylum, the abundance of Helicobacteraceae, which has a carcinogenic effect, was substantially increased in FXR KO mice, and VSL\#3 was able to reduce it (Figure 7B) (27).

At the genus level, lack of FXR increased the abundance of Bacteroides, Coprobacter, Parabacteroides, and Odoribacter of which all were under the Bacteroidetes phylum, and 


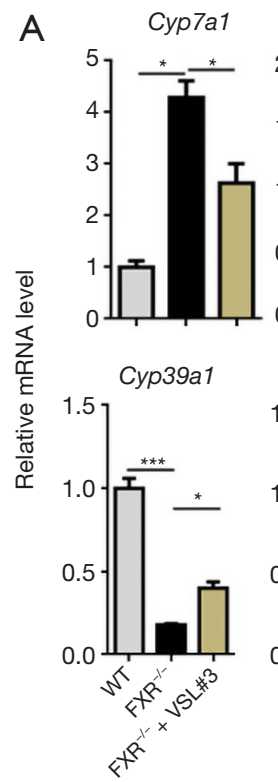

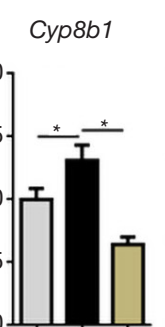
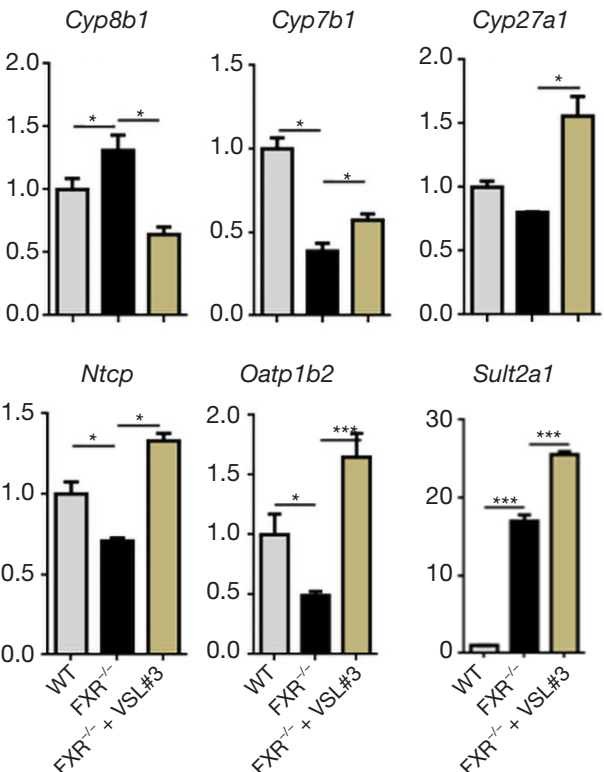

Oatp1b2
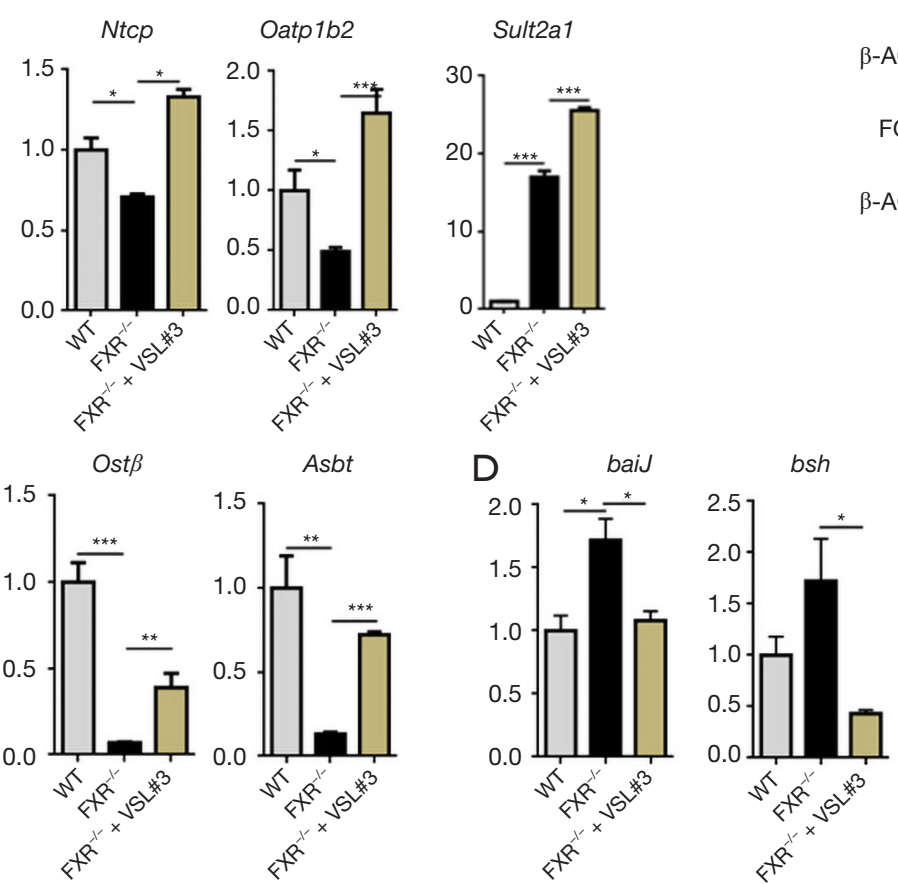

B

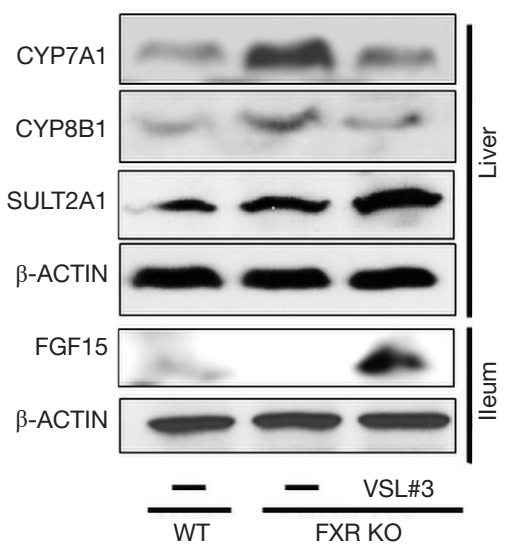

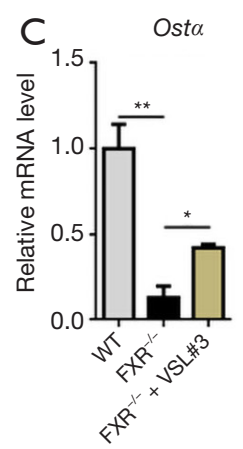

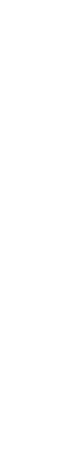

Figure 5 The effect of VSL\#3 on the expression of genes and proteins implicated in BA homeostasis. (A) Hepatic gene expression; (B) Western blot analysis of hepatic and ileal proteins; (C) ileal gene expression; (D) quantitative PCR analyses of microbial genes. Data are expressed as mean $\pm \mathrm{SD} . \mathrm{n} \geq 6$ per group. ${ }^{*}, \mathrm{P}<0.05,{ }^{* *}, \mathrm{P}<0.01$, ${ }^{* * *}, \mathrm{P}<0.001$. BA, bile acid.

VSL\#3 reversed these changes (Figure $7 C$ ). In addition, Clostridium IV under the Firmicutes phylum and Helicobacter under the Proteobacteria phylum were increased with FXR deficiency, and VSL\#3 reduced them (Figure 7C). Moreover, VSL\#3 substantially increased the genera Ruminococcus and Faecalibacterium; which were undetectable in FXR KO mice $(28,29)$ (Figure 7C).

\section{Discussion}

Our study shows that VSL\#3 effectively prevented NASH in WD-fed FXR KO mice, which can develop liver cancer when they are 15 months old (11). The beneficial effects are in part due to regulating BA homeostasis and reconstructing the gut microbiota community structure. VSL\#3 has four
Lactobacillus and three Bifidobacterium species. Our previous work showed that $B$. infantis alone or in combination with milk oligosaccharides as synbiotics are also effective in preventing NASH $(17,18)$. It would be of great interest to further dissect the differential effects of these Lactobacillus and Bifidobacterium species in NASH prevention and treatment.

Our published and unpublished data showed that longterm WD consumption (up to 21 months) only leads to steatosis in wild-type mice (8-10). In contrast to wild-type mice, FXR KO mice have NASH and cancer development. Moreover, male WD-fed FXR KO mice had the most serious NASH (9). Thus, it is exciting that VSL\#3 can prevent this serious NASH, which potentially can be developed into cancer. Long-term (9 months) WD intake may generate 


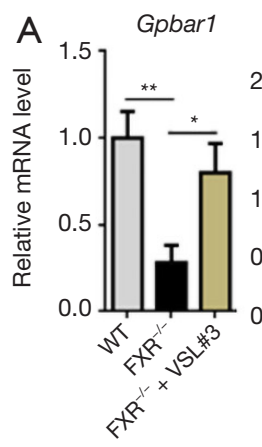

Glp1r
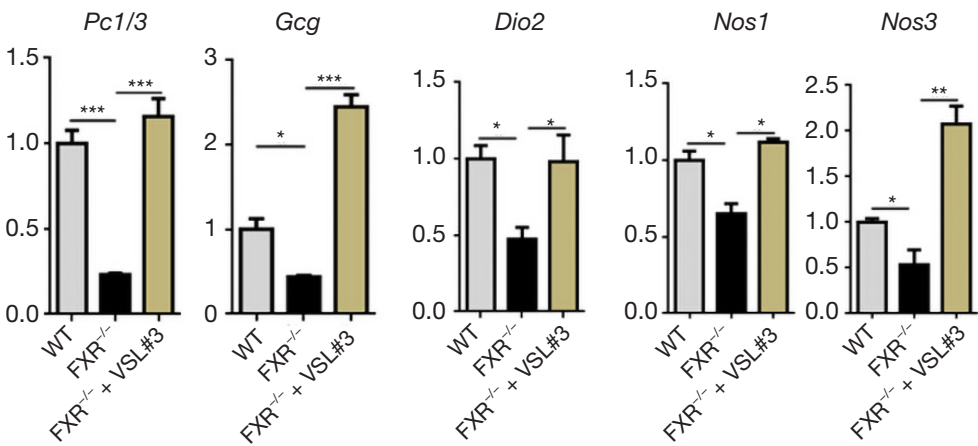

B

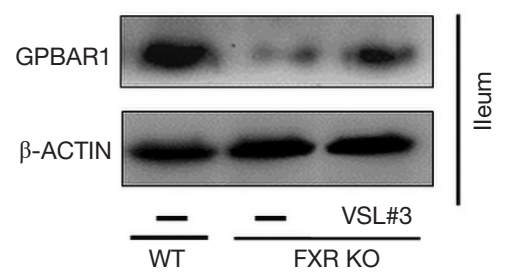

Figure 6 The effects of VSL\#3 on GPBAR1 signaling. Ileal mRNA (A) and protein (B) level of GPBAR1. Data are expressed as mean \pm SD. $\mathrm{n} \geq 6$ per group. ${ }^{*}, \mathrm{P}<0.05,{ }^{* *}, \mathrm{P}<0.01,{ }^{* * *}, \mathrm{P}<0.001$.

oxidative stress and increase ALT. However, VSL\#3 supplementation significantly reduced it. One limitation of the study design was that VSL\#3 was not given to WDfed wild-type mice to study its effect in preventing simple steatosis. However, VSL\#3 did reduce hepatic cholesterol and triglyceride level as well as the steatosis score in WDfed FXR KO mice. In consistency, a 4-month supplement of VSL\#3 also improves NAFLD in children (30).

Human NASH has variable amount of lymphocyte and neutrophil infiltration (31,32). Similarly, WD-fed FXR KO mice had increased hepatic lymphocyte infiltration, and VSL\#3 treatment eliminated it. Our previous work showed that even broad-spectrum coverage antibiotics did not have such effect in WD-fed FXR KO male mice (8). Our data also showed that hepatic and intestinal $I l 1 \beta, I l 6$, Tnfo, and $C c l 17$, which are implicated in hepatitis $\mathrm{C}$ virus infection, alcoholic liver disease, and lipopolysaccharideinduced liver injury, were all reduced by VSL\#3 $(33,34)$. Furthermore, $\operatorname{Reg} 3 \gamma$, which has a protective effect against Gram-positive bacterial infection (35), was reduced in the ileum of FXR KO mice but induced by VSL\#3. Moreover, ileal tight junction genes, which were reduced by FXR KO (13), were induced by VSL\#3, suggesting improved gut permeability. Together, VSL\#3 can be effective in treating liver as well as intestinal inflammation.

The beneficial and detrimental effects of BAs rely on their hydrophilicity, concentration, and duration of exposure (36). Patients with NASH and NAFLD had increased serum and fecal BA levels (37-39). FXR KO mice, which have increased hepatic CYP7A1 and CYP8B1 as well as reduced intestinal FGF15 are constantly exposed to elevated free and secondary BAs $(23,40)$. However, VSL\#3 was able to normalize those changes by reducing hepatic BA synthesizing enzymes CYP7A1 and CYP8B1 as well as increasing intestinal FGF15, thereby leading to reduced BA synthesis through the classical pathway. It has been shown that supplementation of normal chow diet-fed healthy mice with VSL\#3 promotes fecal BA excretion and induces hepatic BA synthesis through inhibiting the gutliver FXR-FGF15 axis (41). Thus, it seems that depending on the metabolic status of the mice, VSL\#3 can either increase or reduce FGF15 signaling, thereby leading to BA homeostasis. In addition, our data suggests that VSL\#3 increased BA synthesis using the alternative pathway, which was in consistency with elevated hepatic and circulating CDCA and LCA. Although the studied mice lack of FXR, those BAs potentially can act through other BA receptors such as GPBAR1, sphingosine-1-phosphate receptor 2, or cholinergic receptor muscarinic 2 to exert their effects $(42,43)$. Additionally, it has been shown that LCA protects hepatocytes from cholestasis-induced liver injury (44). Moreover, LCA is an agonist for pregnane $\mathrm{X}$ receptor and 

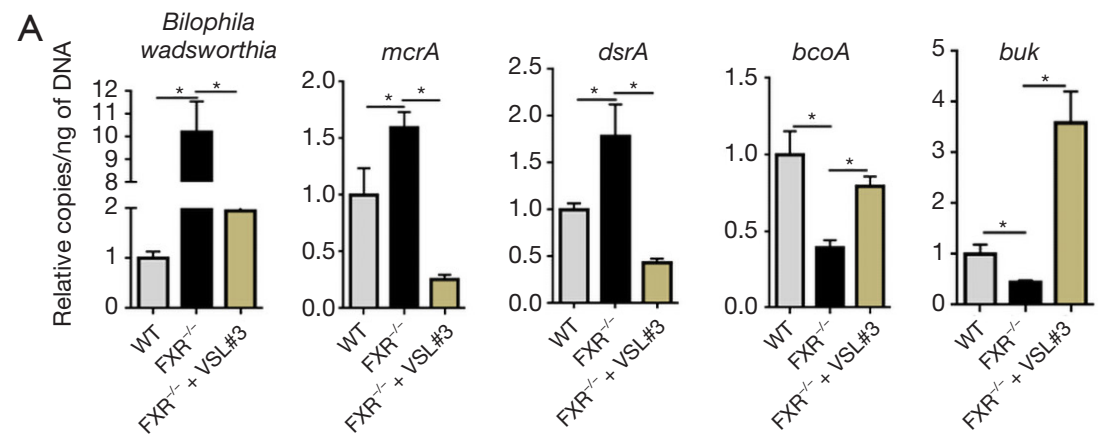

B

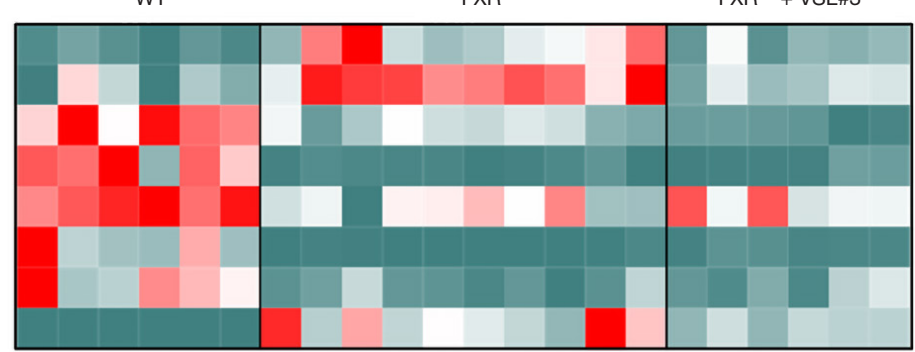

Bacteroidaceae

Porphyromonadaceae

Rikenellaceae

Clostridiaceae

Lachnospiraceae

Erysipelotrichaceae

Coriobacteriaceae

Helicobacteraceae

Low High

C
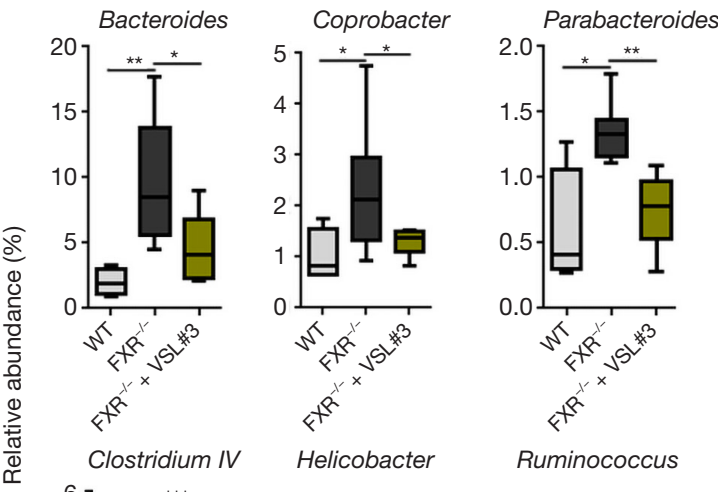

Odoribacter
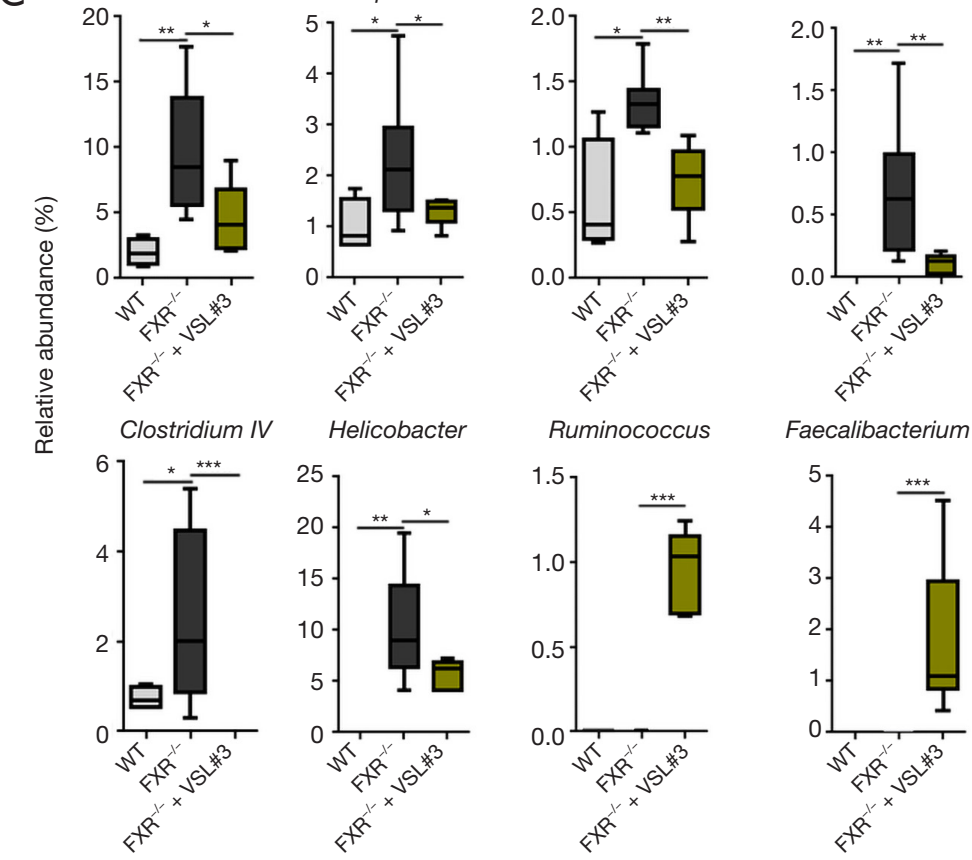

Faecalibacterium

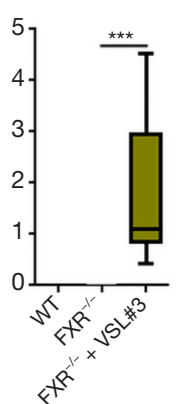

Figure 7 The effect of VSL\#3 in cecal microbiota. (A) Quantitative PCR analysis of microbial genes copy number per ng DNA; (B) heatmap of relative abundance of cecal microbiota at family level; (C) bacteria at genus level. Box plots display the median, 25 th percentile, and 75 th percentile; whiskers display minimum and maximum values. $\mathrm{n} \geq 6$ per group. ${ }^{*}, \mathrm{P}<0.05$, ${ }^{* *}, \mathrm{P}<0.01,{ }^{* * *}, \mathrm{P}<0.001$. 
vitamin $\mathrm{D}$ receptor $(45,46)$. PXR activation induces $\mathrm{BA}$ specific sulfotransferase 2 (SULT2) $(24,47)$, and VSL\#3fed mice had elevated hepatic Sult2a1 mRNA, suggesting increased sulfonation and detoxification.

It has been shown that UDCA is useful in treating rodent and human NASH $(48,49)$. TLCA has a high binding affinity to GPBAR1. GPBAR1 activation reduces diet-induced steatosis and improves insulin sensitivity (24). Moreover, activated GPBAR1 improves intestinal barrier and reduces inflammation (50). Our data revealed that VSL\#3 increased ileal GPBAR1 and induced GPBAR1regulated signaling genes Glp1r, Pc1/3, Gcg, Dio2, Nos1, and Nos 3 , all of which were reduced with FXR inactivation. GPBAR1 signaling gene proprotein convertase $1(P c 1 / 3)$ is responsible for the first step of biosynthesis of insulin and converting glucagon to GLP-1 in intestinal L-cells (51). Activation of the GPBAR1 receptor also reprograms pancreatic $\alpha$ cell to make and secrete GLP-1 locally and promotes $\beta$ cell proliferation and glucose-stimulated insulin secretion $(52,53)$. In addition, GPBAR1 also induces the expression of Dio2, which generates thyroid hormone to stimulate energy metabolism (54). Taken together, VSL\#3 supplementation increased hepatic LCA and TLCA, the endogenous ligands for GPBAR1 and induced Nos3, which may lead to nitric oxide production and protect the liver from endothelial oxidative stress (55).

Dysbiosis leads to hepatic inflammation and carcinogenesis in FXR KO mice (8-10). VSL\#3 reduced the abundance of Bacteroidaceae and Porphyromonadaceae, both of which bloomed in FXR KO mice. The genus Bacteroides was enriched in FXR KO mice but reduced in VSL\#3-fed mice. It has been shown that Bacteroides species are BA-resistant pathogens, which are found in most anaerobic infections with a mortality of $>19 \%$ (56). Moreover, Bacteroides as well as hepatic inflammation persisted even after broad-spectrum antibiotic treatment of WD-fed FXR KO mice (8). One of the most striking differences due to FXR inactivation was the substantial bloom of Helicobacteraceae, which was reduced by VSL\#3 intake. Helicobacter pylorus is well-known for its carcinogenic role (27).

The abundance of Lachnospiraceae is tightly associated with hepatic inflammation. Lachnospiraceae can generate butyrate, which is effective in treating NASH $(10,57,58)$. Moreover, under the Clostridia class, genera Ruminococcus and Faecalibacterium, which were increased by VSL\#3, also produce butyrate $(10,29)$. In consistency with the bacterial abundance, the copy number of butyrate-generating genes was also increased by VSL\#3 supplementation. Our previous publication reveals the beneficial effect of butyrate in preventing NASH in WD-fed FXR KO mice (10). Moreover, VSL\#3 also reduced $\operatorname{ds} A$ and $m c r A$, which generate hydrogen sulfite and methane, respectively that induce DNA damage or inflammation (59).

\section{Conclusions}

In conclusion, VSL\#3 prevents NASH. Hepatic steatosis, inflammation, and insulin sensitivity are all improved by VSL\#3 in WD-fed FXR KO mouse model. The data presented also uncovered the effect of VSL\#3 by establishing eubiosis and BA homeostasis to reverse metabolic disorders.

\section{Acknowledgments}

Funding: This study supported by grants funded by National Institutes of Health CA179582 and CA222490.

\section{Footnote}

Provenance and Peer Review: This article was commissioned by the Guest Editor (Yu-Jui Yvonne Wan) for the series "Gut Microbiome and Liver Disease" published in Hepatobiliary Surgery and Nutrition. The article was sent for external peer review organized by the Guest Editor and the editorial office.

Conflicts of Interest: All authors have completed the ICMJE uniform disclosure form (available at https://hbsn.amegroups. com/article/view/10.21037/hbsn.2019.09.07/coif). YYW serves as the unpaid editorial board member of Hepatobiliary Surgery and Nutrition and served as the unpaid Guest Editor of the series. The other authors have no other conflicts of interest to declare. The series "Gut Microbiome and Liver Disease" was commissioned by the editorial office without any funding or sponsorship.

Ethical Statement: The authors are accountable for all aspects of the work in ensuring that questions related to the accuracy or integrity of any part of the work are appropriately investigated and resolved. Experiments were conducted in accordance with the National Institutes of Health Guidelines for the Care and Use of Laboratory Animals under protocols approved by the Institutional Animal Care and Use Committee of the University of California, Davis. 
Open Access Statement: This is an Open Access article distributed in accordance with the Creative Commons Attribution-NonCommercial-NoDerivs 4.0 International License (CC BY-NC-ND 4.0), which permits the noncommercial replication and distribution of the article with the strict proviso that no changes or edits are made and the original work is properly cited (including links to both the formal publication through the relevant DOI and the license). See: https://creativecommons.org/licenses/by-nc$\mathrm{nd} / 4.0 /$.

\section{References}

1. Bibiloni R, Fedorak RN, Tannock GW, et al. VSL\#3 probiotic-mixture induces remission in patients with active ulcerative colitis. Am J Gastroenterol 2005;100:1539-46.

2. Mencarelli A, Cipriani S, Renga B, et al. VSL\#3 resets insulin signaling and protects against NASH and atherosclerosis in a model of genetic dyslipidemia and intestinal inflammation. PLoS One 2012;7:e45425.

3. Esposito E, Iacono A, Bianco G, et al. Probiotics reduce the inflammatory response induced by a high-fat diet in the liver of young rats. J Nutr 2009;139:905-11.

4. Li Z, Yang S, Lin H, et al. Probiotics and antibodies to TNF inhibit inflammatory activity and improve nonalcoholic fatty liver disease. Hepatology 2003;37:343-50.

5. Velayudham A, Dolganiuc A, Ellis M, et al. VSL\#3 probiotic treatment attenuates fibrosis without changes in steatohepatitis in a diet-induced nonalcoholic steatohepatitis model in mice. Hepatology 2009;49:989-97.

6. Dashti HM, Mathew TC, Hussein T, et al. Long-term effects of a ketogenic diet in obese patients. Exp Clin Cardiol 2004;9:200-5.

7. Rinella ME, Green RM. The methionine-choline deficient dietary model of steatohepatitis does not exhibit insulin resistance. J Hepatol 2004;40:47-51.

8. Sheng L, Jena PK, Liu HX, et al. Gender Differences in Bile Acids and Microbiota in Relationship with Gender Dissimilarity in Steatosis Induced by Diet and FXR Inactivation. Sci Rep 2017;7:1748.

9. Jena PK, Sheng L, Liu HX, et al. Western Diet-Induced Dysbiosis in Farnesoid X Receptor Knockout Mice Causes Persistent Hepatic Inflammation after Antibiotic Treatment. Am J Pathol 2017;187:1800-13.

10. Sheng L, Jena PK, Hu Y, et al. Hepatic inflammation caused by dysregulated bile acid synthesis is reversible by butyrate supplementation. J Pathol 2017;243:431-41.

11. Yang F, Huang X, Yi T, et al. Spontaneous development of liver tumors in the absence of the bile acid receptor farnesoid X receptor. Cancer Res 2007;67:863-7.

12. Su H, Ma C, Liu J, et al. Downregulation of nuclear receptor FXR is associated with multiple malignant clinicopathological characteristics in human hepatocellular carcinoma. Am J Physiol Gastrointest Liver Physiol 2012;303:G1245-53.

13. Sinal CJ, Tohkin M, Miyata M, et al. Targeted disruption of the nuclear receptor FXR/BAR impairs bile acid and lipid homeostasis. Cell 2000;102:731-44.

14. Jena PK, Sheng L, Di Lucente J, et al. Dysregulated bile acid synthesis and dysbiosis are implicated in Western diet-induced systemic inflammation, microglial activation, and reduced neuroplasticity. FASEB J 2018;32:2866-77.

15. Liu HX, Rocha CS, Dandekar S, et al. Functional analysis of the relationship between intestinal microbiota and the expression of hepatic genes and pathways during the course of liver regeneration. J Hepatol 2016;64:641-50.

16. O'Keefe SJ, Li JV, Lahti L, et al. Fat, fibre and cancer risk in African Americans and rural Africans. Nat Commun 2015;6:6342.

17. Jena PK, Sheng L, Nagar N, et al. Synbiotics Bifidobacterium infantis and milk oligosaccharides are effective in reversing cancer-prone nonalcoholic steatohepatitis using western diet-fed FXR knockout mouse models. J Nutr Biochem 2018;57:246-54.

18. Jena PK, Sheng L, Nagar N, et al. The effect of synbiotics Bifidobacterium infantis and milk oligosaccharides on shaping gut microbiota community structure and NASH treatment. Data Brief 2018;19:1025-9.

19. Ou J, Carbonero F, Zoetendal EG, et al. Diet, microbiota, and microbial metabolites in colon cancer risk in rural Africans and African Americans. Am J Clin Nutr 2013;98:111-20.

20. Williams JE, Carrothers JM, Lackey KA, et al. Human Milk Microbial Community Structure Is Relatively Stable and Related to Variations in Macronutrient and Micronutrient Intakes in Healthy Lactating Women. J Nutr 2017;147:1739-48.

21. Sheng L, Jena PK, Liu HX, et al. Obesity treatment by epigallocatechin-3-gallate-regulated bile acid signaling and its enriched Akkermansia muciniphila. FASEB J 2018. [Epub ahead of print].

22. Wang Q, Garrity GM, Tiedje JM, et al. Naïve Bayesian 
Classifier for Rapid Assignment of rRNA Sequences into the New Bacterial Taxonomy. 2007;73:5261-7.

23. Meng Z, Wang Y, Wang L, et al. FXR regulates liver repair after CCl4-induced toxic injury. Mol Endocrinol 2010;24:886-97.

24. Pathak P, Liu H, Boehme S, et al. Farnesoid X receptor induces Takeda G-protein receptor 5 cross-talk to regulate bile acid synthesis and hepatic metabolism. J Biol Chem 2017;292:11055-69.

25. Martinez I, Wallace G, Zhang C, et al. Diet-induced metabolic improvements in a hamster model of hypercholesterolemia are strongly linked to alterations of the gut microbiota. Appl Environ Microbiol 2009;75:4175-84.

26. Martínez I, Lattimer JM, Hubach KL, et al. Gut microbiome composition is linked to whole grain-induced immunological improvements. ISME J 2013;7:269-80.

27. Nambiar PR, Kirchain S, Fox JG. Gastritis-associated adenocarcinoma and intestinal metaplasia in a Syrian hamster naturally infected with Helicobacter species. Vet Pathol 2005;42:386-90.

28. Takahashi K, Nishida A, Fujimoto T, et al. Reduced Abundance of Butyrate-Producing Bacteria Species in the Fecal Microbial Community in Crohn's Disease. Digestion 2016;93:59-65.

29. Zhou L, Zhang M, Wang Y, et al. Faecalibacterium prausnitzii Produces Butyrate to Maintain Th17/ Treg Balance and to Ameliorate Colorectal Colitis by Inhibiting Histone Deacetylase 1. Inflamm Bowel Dis 2018;24:1926-40.

30. Alisi A, Bedogni G, Baviera G, et al. Randomised clinical trial: The beneficial effects of VSL\#3 in obese children with non-alcoholic steatohepatitis. Aliment Pharmacol Ther 2014;39:1276-85.

31. Gadd VL, Skoien R, Powell EE, et al. The portal inflammatory infiltrate and ductular reaction in human nonalcoholic fatty liver disease. Hepatology 2014;59:1393-405.

32. Alkhouri N, Morris-Stiff G, Campbell C, et al. Neutrophil to lymphocyte ratio: a new marker for predicting steatohepatitis and fibrosis in patients with nonalcoholic fatty liver disease. Liver Int 2012;32:297-302.

33. Heiseke AF, Faul AC, Lehr HA, et al. CCL17 promotes intestinal inflammation in mice and counteracts regulatory $\mathrm{T}$ cell-mediated protection from colitis. Gastroenterology 2012;142:335-45.

34. Marra F, Tacke F. Roles for chemokines in liver disease. Gastroenterology 2014;147:577-94.e1.
35. Loonen LM, Stolte EH, Jaklofsky MT, et al. REG3 gamma-deficient mice have altered mucus distribution and increased mucosal inflammatory responses to the microbiota and enteric pathogens in the ileum. Mucosal Immunol 2014;7:939-47.

36. Tsuei J, Chau T, Mills D, et al. Bile acid dysregulation, gut dysbiosis, and gastrointestinal cancer. Exp Biol Med (Maywood) 2014;239:1489-504.

37. Mouzaki M, Wang AY, Bandsma R, et al. Bile Acids and Dysbiosis in Non-Alcoholic Fatty Liver Disease. PLoS One 2016;11:e0151829.

38. Jiao N, Baker SS, Chapa-Rodriguez A, et al. Suppressed hepatic bile acid signalling despite elevated production of primary and secondary bile acids in NAFLD. Gut 2018;67:1881-91.

39. Chiang JYL. Targeting bile acids and lipotoxicity for NASH treatment. Hepatol Commun 2017;1:1002-4.

40. Chiang JY. Bile acid metabolism and signaling. Compr Physiol 2013;3:1191-212.

41. Degirolamo C, Rainaldi S, Bovenga F, et al. Microbiota modification with probiotics induces hepatic bile acid synthesis via downregulation of the Fxr-Fgf15 axis in mice. Cell Rep 2014;7:12-8.

42. Wan YJY, Sheng L. Regulation of bile acid receptor activity. Liver Res 2018;2:180-5.

43. Jena PK, Sheng L, McNeil K, et al. Long-term Western diet intake leads to dysregulated bile acid signaling and dermatitis with Th2 and Th17 pathway features in mice. J Dermatol Sci 2019;95:13-20.

44. Han S, Li T, Ellis E, et al. A novel bile acid-activated vitamin D receptor signaling in human hepatocytes. Mol Endocrinol 2010;24:1151-64.

45. Adachi R, Honma Y, Masuno H, et al. Selective activation of vitamin $\mathrm{D}$ receptor by lithocholic acid acetate, a bile acid derivative. J Lipid Res 2005;46:46-57.

46. Staudinger JL, Goodwin B, Jones SA, et al. The nuclear receptor PXR is a lithocholic acid sensor that protects against liver toxicity. Proc Natl Acad Sci U S A 2001;98:3369-74.

47. Echchgadda I, Song CS, Oh T, et al. The xenobioticsensing nuclear receptors pregnane $\mathrm{X}$ receptor, constitutive androstane receptor, and orphan nuclear receptor hepatocyte nuclear factor 4alpha in the regulation of human steroid-/bile acid-sulfotransferase. Mol Endocrinol 2007;21:2099-111.

48. Tsuchida T, Shiraishi M, Ohta T, et al. Ursodeoxycholic acid improves insulin sensitivity and hepatic steatosis by inducing the excretion of hepatic lipids in high-fat diet- 
fed KK-Ay mice. Metabolism 2012;61:944-53.

49. Xiang Z, Chen YP, Ma KF, et al. The role of ursodeoxycholic acid in non-alcoholic steatohepatitis: a systematic review. BMC Gastroenterol 2013;13:140.

50. Thomas C, Gioiello A, Noriega L, et al. TGR5-mediated bile acid sensing controls glucose homeostasis. Cell Metab 2009;10:167-77.

51. Steiner DF, Rouille Y, Gong Q, et al. The role of prohormone convertases in insulin biosynthesis: evidence for inherited defects in their action in man and experimental animals. Diabetes Metab 1996;22:94-104.

52. Katsuma S, Hirasawa A, Tsujimoto G. Bile acids promote glucagon-like peptide-1 secretion through TGR5 in a murine enteroendocrine cell line STC-1. Biochem Biophys Res Commun 2005;329:386-90.

53. Kumar DP, Asgharpour A, Mirshahi F, et al. Activation of Transmembrane Bile Acid Receptor TGR5 Modulates Pancreatic Islet alpha Cells to Promote Glucose Homeostasis. J Biol Chem 2016;291:6626-40.

Cite this article as: Jena PK, Sheng L, Li Y, Wan YJY. Probiotics VSL\#3 are effective in reversing non-alcoholic steatohepatitis in a mouse model. HepatoBiliary Surg Nutr 2020;9(2):170-182. doi: 10.21037/hbsn.2019.09.07
54. Watanabe M, Houten SM, Mataki C, et al. Bile acids induce energy expenditure by promoting intracellular thyroid hormone activation. Nature 2006;439:484-9.

55. Keitel V, Reinehr R, Gatsios P, et al. The G-protein coupled bile salt receptor TGR5 is expressed in liver sinusoidal endothelial cells. Hepatology 2007;45:695-704.

56. Wexler HM. Bacteroides: the good, the bad, and the nitty-gritty. Clin Microbiol Rev 2007;20:593-621.

57. Jin CJ, Sellmann C, Engstler AJ, et al. Supplementation of sodium butyrate protects mice from the development of non-alcoholic steatohepatitis (NASH). Br J Nutr 2015;114:1745-55.

58. Vital M, Howe AC, Tiedje JM. Revealing the Bacterial Butyrate Synthesis Pathways by Analyzing (Meta) genomic Data. MBio 2014;5:e00889.

59. Attene-Ramos MS, Wagner ED, Gaskins HR, et al. Hydrogen sulfide induces direct radical-associated DNA damage. Mol Cancer Res 2007;5:455-9. 